\title{
Hybrid Sport Configurations: The Intertwining of the Physical and the Digital
}

\author{
Lucas Goebeler \\ Copenhagen Business School \\ lgo.digi@cbs.dk
}

\author{
Willem Standaert \\ HEC Liège - University of Liège \\ willem.standaert $@$,uliege.be
}

\author{
Xiao Xiao \\ Copenhagen Business School \\ xx.digi@cbs.dk
}

\begin{abstract}
The motivation for this research follows from our observation of the increasing influence of digitalization on sporting activities and the emergence of physicaldigital hybrid sport. While traditional, physical sport gradually embraces digital elements and experiences to the game, born-digital eSport increasingly involves physical elements in its setting (e.g., offline tournaments). In this paper, we investigate various physical-digital hybrid configurations of existing and emerging sporting activities and their implications for the fusing of the digital and physical worlds. Based on an inductive approach and drawing from existing literature on physical-digital hybridity, we conceptualize four sport clusters (digitally supported sport, digitally augmented sport, digitally replicated sport, and digitally translated sport) along three dimensions: the sporting activities (especially in terms of the relationship between the digital and physical components), the sporting arena, and actors' influence. Based on our conceptualization and observations, we discuss implications for both the information systems and sport management domains.
\end{abstract}

\section{Introduction}

Sport, as one of the oldest traditions and activities carried out by human beings, is traditionally understood to be comprised of physical activities [5, 21]. Now we are observing how digital technologies are gradually shaping and changing the face of sport in how the sporting activities are prepared and carried out $[6,7,24,43]$. While prior technological innovation in sport was often engineering-driven and centralized [23, 38 ], the use of digital technology potentially renders sport-related innovation distributed, co-created, and generative in nature [27, 47]. At the same time, the increasing popularity of eSport has triggered debates on whether eSport should be considered sport both in academia [5] and in practice [8]. No matter the stance, this sporting activity born in the digital realm has prompted scholars and practitioners to rethink the nature of sport $[5,14,16]$.
The increasing permeation of digital technologies in traditional (physical) sport and the growing power of eSport put forward the idea that we are entering a new sport era, where sport does not just take place in the physical world but also in the digital realm.

One example of such physical-digital hybrid sport can be found in Formula E, the first fully electric motorsport series. Besides its emphasis on sustainability, Formula E has also pioneered the use of digital technologies to introduce new activities that have a direct impact on the physical performance [17]. More specifically, through FanBoost, fans can vote online for their favorite driver, the most popular of whom will then be awarded with an extra speed boost in the race. Such sporting activity is digitally facilitated and is enabled by the embedded computing capacity of the physical device (in this case, the racing car). This also creates a new dynamic in the competition itself as fan engagement is directly influencing the in-game activities. Another hybrid sport example is virtual bicycle racing, such as the 2020 Virtual Tour de France [1]. Following the lockdown measures during the Covid-19 pandemic, virtual cycling races became mainstream as professional cyclists, some of which confined to their apartment, were eager to compete virtually. In this case, the digital technology captures the physical activity through sensors on the bike station and then enables a competition of identical or similar nature in the virtual space. The manifestation of digital technologies in physical contexts and of physical activities in digital contexts, as illustrated in these examples, reflects the "necessity" to discuss the physical aspects of digital innovation [46:12].

Against the backdrop of its inherent physicality, the emerging and increasingly digital configurations of sport provide a valuable context to further investigate the hybrid nature of digital innovation. This initial study strives to conceptualize the influence of digital technologies on sporting activities, by focusing on sport with both digital and physical components. More specifically, we strive to answer this research question: how can different configurations of physical-digital hybrid sport be conceptualized? 
We introduce the concept of physical-digital hybrid sport, in which the boundaries between what is physical and what is digital become blurred [25, 28]. Such hybridity, going beyond the mere coexistence of physical and digital, can be observed in the activities of the athletes [24], the role of other actors, such as the fans [17], and the 'arena' or context in which the sporting activities take place [33].

We believe that our conceptualization of hybridity in sport can serve a number of purposes. First, the sport industry provides a fertile ground to further understand a hybrid artifact/activity as well as to study the importance of physicality in digital innovation, as called for by IS scholars [3, 46]. It can therefore serve as a setting to observe and conceptualize different forms of hybridity that can be useful or applicable for other contexts. Second, revisiting and conceptualizing the nature of sport in the digital era, when digital technologies have permeated every aspect of our lives [45], will help us understand the continuous evolution of sport. Moreover, it can help sport organizations to better manage and "skate to where the puck is going to be, not where it has been."

\section{Literature review}

Scholars in sport management have defined sport as a physical activity that requires skill, is competitive in nature, and has a level of stability in its set-up and rules [5]. A key element that differentiates sport from games is the focus on competition [21] that revolves around testing a specific physical capability of human beings [36]. Further, the rules, procedures, and activities around such competition are set and stable, which speak to sport as a form of organization with its own institutional settings [5]. Therefore, existing research on sport in social science mostly studies such organizational and institutional nature of sport in understanding how sport organizations, as a specific and different type of business, conduct themselves in operations and management.

\subsection{Digitalizing traditional sport}

On the topic of sport and digital technologies, existing research, both in sport management and in information systems, has focused on social media for fan engagement, data analytics for athlete recruitment and performance enhancement, and to a lesser extent, the facilitation of sport and sport business through new technologies. In terms of social media, studies have

\footnotetext{
${ }^{1}$ Quote often attributed to ice hockey player Wayne Gretzky.
}

been conducted to understand how individual players and sport organizations utilize social media to build identities and manage brands [2, 12], what drives sport consumers to engage with sport organizations through social media [20,35], and how social media reflect and change the media landscape and power dynamics in sport [44]. On the data analytics side, research in IS has set out to understand the adoption of data analytics among sport organizations $[6,39]$, the value creation of data analytics for sport organizations [4, 37], and the design of sport-oriented analytics platforms [30]. Additionally, recent studies have addressed the facilitation of sport with digital technologies. Such studies observe the use of emerging technologies such as AR (augmented reality) to engage spectators as participants of the competition [34] or the use of AIbased evaluation systems in judged sports [7].

However, what should be noted is that research in this area is still in its nascent stage [13] and many of the studies, especially those in the IS domain, simply approach the phenomenon with the sport industry as yet another business context [43].

\subsection{Born-digital sport}

With the rise and increasing popularity of eSport, a new research stream has emerged. Research on eSport revolves around the debate on the nature of eSport and whether it should be regarded as sport at all. Wagner [41] defined eSport as "an area of sport activities in which people develop and train mental or physical abilities in the use of information and communication technologies" (p. 182). He argues that the competition element in eSport makes it "sporty" and separates it from other video games. Similarly, other scholars point out that eSport possesses the element of physicality [16], in its requirement of fine motor movement that is measured by parameters such as the number of actions per minute [11].

On the other side of the aisle, Wright [42] argues that the physical activity in sport is one that cannot be undertaken at a distance and that distributed activities should therefore not be considered sport. Furthermore, Hallmann and Giel [14] argue that the lack of formal and stable organizational structure determines that eSport cannot be considered sport yet. Regardless of such debate, what is in consensus is the potential synergy between eSport and traditional sport for marketing [29] and fan engagement purposes [5].

In this study, we take the stand in recognizing the sport nature of eSport. As such, they represent a borndigital sport, i.e. sport performed mainly by digital means and competing on a digital platform. 


\subsection{A concept of physical-digital hybridity}

By recognizing the sport nature of eSport, we need to address how the traditional physical and the emerging digital elements of sport encounter each other. One way to think of instances of such configurations is physical-digital hybrids. The concept of physical-digital hybridity has been considerably used to refer to combined material and digital artifacts $[3,9,10]$ that involve micro-level encounters of loosely coupled physical and digital components [45]. Our understanding of hybridity in the context of this study departs from such micro-level conceptualization of relationships between software and hardware central to hybrid digital artifacts. Instead, we relate to the concept on a systemic or macro level, with activities involving either physical or virtual interactions between people and/or objects [25, 26]. So, while specific activities and objects can be material or digital, we conceptualize not their materiality ${ }^{2}$ [19] but the physicality of their interaction, i.e. whether the interaction takes place physically or is digitally facilitated.

Hybrid configurations do not simply reflect the coexistence of physical and digital components but also their interaction or intertwining relationship [28]. Intertwining, as described by Robey et al. [28], is a powerful metaphor illustrating the mutual involvement of physical and digital elements. Their framework identifies four effects of intertwining relationships: reciprocal, i.e. they are mutually interdependent; strengthened through the reinforcement of one element by another; complementary to each other by compensating each other's weaknesses; interact with one another to create synergy, exceeding the simple combination of the individual elements. The framework also notes that not necessarily all four effects are present equally in every individual activity.

Such an understanding of intertwining relationships provides an initial basis to categorize and conceptualize the relationship between physical and digital elements in hybrid configurations of sport, as presented in the following sections.

\section{The traditional configuration of sport}

To develop a framework of physical-digital hybrid sport, we first theorize on the dimensions that

\footnotetext{
${ }^{2}$ The Oxford English Dictionary defines materiality as "the quality of being composed of matter" [48], whereas physicality is describes the quality of having a physical presence (perceived through senses) [49].
}

constitute the physical configuration of sport in a traditional sense. As defined above, sport is traditionally characterized as the physical activity performed in the form of an organized competition, which is governed by rules [36]. The organized competition can further be characterized by the spatial and temporal limitations of sport [21], i.e. it occurs in a specific time and place [43] and cannot be undertaken at a distance [42].

It can be synthesized that sport, as traditionally defined, is shaped by the physical activity that is governed by rules and structures (how activities interact with one another), in a physical location (the sporting arena), performed by the human athlete(s) (the actors), resulting in an outcome that is the competitive performance (Figure 1). We suggest the relationships between in-game activities, actors, and sporting arena as dimensions characterizing different configurations of sport. In the remainder of this section, we discuss how these three dimensions apply to traditional, physical sport. In the next sections, we build on these same dimensions to conceptualize how they change through digitalization.

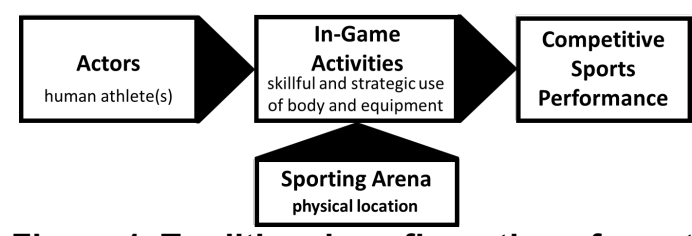

Figure 1. Traditional configuration of sport

\subsection{In-game activities}

To delineate what constitutes a sporting activity, we consider the extent to which activities directly and instantly impact the competitive performance. For instance, an athlete's training or a racing engineer's car preparation activities, do directly influence the resulting performance. However, considering the temporal limitations, i.e. momentary nature, attributed to sport [31], the sport performance happens at a specific time and therefore the result of the competition is based on the performance output during that specific moment in time. We therefore treat activities such as physical and mental training as preparatory activities, in contrast to what can be referred to as in-game activities. Such preparatory activities enhance the likelihood of a strong performance without instantly influencing the specific competitive performance output. On the other hand, fan activities happen in the same temporal limitation, but the impact is not considered direct. Hence, in-game activities are mostly limited to the physical activity performed by an athlete. 
This physical activity refers to or involves an athlete's skillful and strategic use of their body [18]. In some sport, this physical activity involves the athlete potentially interacting with equipment, i.e. a technological component [21] or "everyday artifact" [45], such as a ball, bat, or car. Some supporting activities, such as coaching (e.g. making substitutions) during a competition, can also have direct and instant impact and can be considered as in-game activities.

From these in-game activities, we can further distinguish activities that create the performance from activities that facilitate the competition and performance. Such facilitating activities refer to ones that govern the competition, such as judging and refereeing.

\subsection{Sporting arena}

Considering the spatial and temporal limitations that are attributed to sport [31], the sporting arena can traditionally be considered as the physical location at which sport performance is created and where the competition takes place. Given the above understanding that the sport performance is created from the interactions of the various sporting activities, one could describe the physical arena as the place where the physical interaction of the competitive activities takes place [25].

As outlined in section 3.1., preparatory activities have an impact by increasing the likelihood of a good performance. However, due to the momentary nature of sport, only the in-game activities can be considered to be creating the performance output. Therefore, training facilities, dressing rooms, or health facilities are not considered to be part of the physical arena. Consequently, one could describe the physical arena as encompassing all physical interaction of competitive in-game activities.

Another aspect present in the physical, open-air arena is the unpredictability of environmental influences such as rain, wind, or temperature. These environmental conditions of the physical arena can have a direct impact on an athlete's performance. However, they are generally understood to, at least theoretically, affect everyone equally and are therefore a reinforcement of the strategic unpredictability of sport [31].

\subsection{Actors of sport and their influence}

The various sporting activities are performed by actors. Focusing on the in-game activities that create the sport performance as outlined in the previous section, we can make several distinctions to classify the various actors involved, as well as the influence they have on the performance.

Firstly, we can make a distinction of actors along the line of competitive and facilitating activities (see Section 3.1). Competitive actors are the ones performing the competitive activities. This can be the individual athlete performing a long jump or it can be a football team consisting of a number of athletes interacting with each other to create their team's performance. Non-competitive actors in contrast perform the facilitating activities. This includes actors such as judges, referees, or stewards.

Secondly, among the competitive actors we can observe three orders of proximity to the performed physical core activity. First order actors are closest in proximity and represent the athletes and teams themselves who are actively performing the physical activity. By definition, athletes exert a direct and instant physical influence on the sporting activity in question. Second order actors are in close proximity to the performed core activity without physically performing it themselves. They are often performing preparatory activities but can also engage in in-game supporting activities. Second order actors engaging in such supporting activities have mostly cognitive influence on the core activity through, for example, ingame strategizing in the case of coaches or team principles. Lastly, third order actors refer to the spectators, who are generally separated from the performance of the core activity. The influence of third order actors is, as suggested by practitioners, mostly an emotional influence [15] rather than a physical or cognitive influence. In academia the actual performance impact of fans is under debate with studies finding significant [32] as well as insignificant impacts [40].

\section{Conceptualization of the hybrid configuration of sport}

In this section, we present our categorization of physical-digital hybrid sport configurations. The traditional configuration of sport on the one hand and eSport on the other hand, represent the two extremes of a continuum of sport configurations, with the traditional sport mostly involving physical elements while eSport as born digital. In between these extremes, we place hybrid configurations of sport, where the fusing of physical and digital can be observed $[3,45]$. 
Table 1. Four clusters of physical-digital hybrid sport configurations

\begin{tabular}{|c|c|c|c|c|}
\hline & & & In-Game Activities & Actors involvement and influence \\
\hline \multirow{4}{*}{ 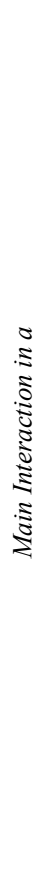 } & \multirow[b]{2}{*}{ 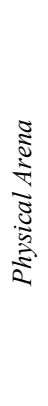 } & $\begin{array}{l}\text { Digitally } \\
\text { supported } \\
\text { sport }\end{array}$ & $\begin{array}{l}\text { - The performance outcome is a direct result of a physical human input. } \\
\text { - Position of the digital component: peripheral, outside competition } \\
\text { - Role of the digital component: supporting but not altering } \\
\text { competition (e.g. non-competitive actors; preparatory activities); }\end{array}$ & $\begin{array}{l}-1^{\text {st }} \text { order: physical performance } \\
-2^{\text {nd }} \text { order: cognitive influence } \\
-3^{\text {rd }} \text { order: no or emotional influence }\end{array}$ \\
\hline & & $\begin{array}{l}\text { Digitally } \\
\text { augmented } \\
\text { sport }\end{array}$ & $\begin{array}{l}\text { - The performance outcome is dependent on a combination of physical } \\
\text { and digital inputs. A physical, often equipment-based, output is } \\
\text { captured digitally and augmented through a digitally mediated } \\
\text { feedback loop } \\
\text { - Position of the digital component: embedded in equipment as integral } \\
\text { part of the competition } \\
\text { - Role of the digital component: capturing performance and enabling } \\
\text { live feedback loops }\end{array}$ & $\begin{array}{l}\text { - } 1^{\text {st }} \text { order: physical performance } \\
-2^{\text {nd }} \text { order: cognitive and physical } \\
\text { influence } \\
-3^{\text {rd }} \text { order: emotional and physical } \\
\text { influence }\end{array}$ \\
\hline & \multirow{2}{*}{ 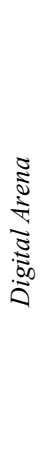 } & $\begin{array}{l}\text { Digitally } \\
\text { replicated } \\
\text { sport }\end{array}$ & $\begin{array}{l}\text { - The performance outcome is generated from a physical input that is } \\
\text { directly }(1: 1) \text { replicated in a digital arena. The } 1: 1 \text { relationship leads to } \\
\text { one actor controlling only one avatar. } \\
\text { - Position of the digital component: becomes the location of the } \\
\text { competition } \\
\text { - Role of the digital component: full mediation of the physical input } \\
\text { while maintaining the original nature; }\end{array}$ & $\begin{array}{l}-1^{\text {st }} \text { order: physical performance and } \\
\text { digital skill/knowledge } \\
-2^{\text {nd }} \text { order: cognitive influence } \\
-3^{\text {rd }} \text { order: non-competitive } \\
\text { participation through digital } \\
\text { engagement }\end{array}$ \\
\hline & & $\begin{array}{l}\text { Digitally } \\
\text { translated } \\
\text { sport }\end{array}$ & $\begin{array}{l}\text { - The performance outcome is realized through the translation of a } \\
\text { physical input into a virtual activity of different nature. One input can } \\
\text { lead to many outputs, i.e. a player can control one or more avatars. } \\
\text { - Position of the digital component is the competition itself } \\
\text { - Role of the digital component: translating, realizing, and governing } \\
\text { the performance; translation of the physical input, radically changing } \\
\text { the original competitive nature }\end{array}$ & $\begin{array}{l}-1^{\text {st }} \text { order: physical performance and } \\
\text { digital skill/knowledge } \\
-2^{\text {nd }} \text { order: cognitive and potentially } \\
\text { digital influence } \\
-3^{\text {rd }} \text { order: potential digital influence; } \\
\text { non-competitive participation through } \\
\text { digital engagement }\end{array}$ \\
\hline
\end{tabular}

We would like to emphasize that (1) such hybrid configurations are more than just the combination of the individual components, but result from the intertwining relationship of the physical and the digital components [28]; (2) with increasing digitalization, we observe a "move to the middle". In other words, all sport can be conceptualized as being hybrid in nature.

Our conceptualization of the hybrid configuration of sport is based on inductive reasoning. First, we collected evidence by observing a number of popular sports (among others basketball, football, motorsport, and cycling). We analyzed these sports across the three dimensions identified in section 3 (namely, the sporting activities, the actors involved, and the sporting arena). Second, we further analyzed the sporting activities by differentiating the physical and digital components that produce the sporting activities, as well as conceptualizing the different relationships between the different components. Third, we identified four clusters of configurations that display varying degrees of intertwining: digitally supported sport, digitally augmented sport, digitally replicated sport, and digitally translated sport (see Table 1).

In the next sections, we present and discuss the identified four clusters. The main interaction for the first two clusters is physical and located in the physical arena. In contrast, for the latter two clusters, the main interaction is facilitated digitally. They are carried out in a digital space that we refer to as the digital arena.

\subsection{Digitally supported sport}

Digitally supported sport includes most of the traditional sport, such as soccer, basketball, tennis, etc. In this cluster, digitalization is observed outside of the in-game activities. The competition remains as a direct physical output from a pure physical input. To put it differently, the digital component is not part of the competitive interaction and is playing a peripheral, supporting role. Hence, digital components in this cluster affect primarily facilitating or preparatory activities. Examples include technologies such as Hawkeye or VAR technologies implemented to improve the refereeing quality, $\mathrm{A} / \mathrm{VR}$ assisted training sessions to improve the quality of athlete preparation, and the use of data analytics to assist the strategizing and cognitive decision making of coaches.

Central to the direct and physical relationship between input activities and performance output are the physical interactions taking place in a physical arena, such as a football stadium. This is in part due to the sport being set up around direct interactions of the human athletes, that are not and cannot be facilitated through digital technology.

This form of relationship and interaction does not afford the facilitation of second and third order actors' influence on the core activity. Therefore, the main actors that produce the sport performance are the athletes (or first order actors). 
In this cluster, despite the increasing presence of digital technologies, the core of the competition remains the competition of physical prowess.

\subsection{Digitally augmented sport}

The second cluster we have identified is digitally augmented sport. Examples of such sport include modern motorsport. In this cluster, physical input (e.g., footing the speed paddle) generates physical output (e.g., racing speed). However, this physical output is generated through equipment (e.g. the racing car). In the example of motorsport, this equipment-based relationship allows for the augmentation of the performance output by digitally capturing the physical input in real-time, feeding it to a team of engineers who can analyze the data, provide live feedback, and adjust car settings remotely. Such a feedback loop is digitally mediated (through the electronic control unit built in the car), and hence the performance is dependent on physical input by the driver and digital input by the team engineer/principle/strategist. This shows that the digital components are embedded as integral parts of the competition. Another example of such embedded digital components is FanBoost in Formula E (as introduced above), in which the digitally facilitated engagement of spectators unlocks additional energy for the driver.

Hence, in this cluster of sport configuration, while the main activity is performed in a physical arena (e.g., the racetrack), the digital capabilities, embedded in equipment, allow for the potential augmentation of performance. Characteristic to this cluster is that the computational capabilities embedded in and connected to sporting equipment facilitate an opening for second (e.g., remote access to the car) and third order actors (e.g., FanBoost) to have a physical influence on the core activity performed by the athlete.

The difference between digitally augmented sport and the previous cluster of digitally supported sport is that the competition does not only concern the physical skills (e.g., driving skill in motorsports) but also the digital skills (e.g., how well the team analyzes and acts on the data) and, in some cases, popularity of the team and drivers (e.g., FanBoost). However, the competition can still carry on without the digital components (see Formula One in its old days), which makes it similar to a digitally supported sport while distinguishing it from the following two clusters.

\subsection{Digitally replicated sport}

Third, there is the cluster of digitally replicated sport, where for example virtual bike races are located. In this cluster, the digital component represents the one-to-one replication of the physical counterpart. Correspondingly, the sport performance depends on physical inputs, which are fully mediated through digital means and replicated in a digital arena. For instance, as a reaction to the pandemic restrictions, a shortened version of the Tour de France was carried out in such a way [1]. All participants were equipped with an internet-connected stationary bicycle. The bicycling activities performed on such station are then replicated in the virtual sphere, in which the real landscape and environment, such as the iconic Champs Élysées finish line in Paris, are simulated. The digital arena is also captured and displayed on the screen attached to the station, from which the participants can observe and monitor the situation of the competition (e.g., upcoming climbs, position in the competition). In this sense, the core of the competition (or what the competition is based on) remains unchanged (the physical capability, e.g., athletes' cycling skills). However, some skills become less important in the digital arena, for instance the ability to skillfully ride over cobbles. Similarly, the digital nature of the arena may require some additional skills/knowledge from the players (e.g., perceiving competitors position and activities based on the on-screen avatar), which are different from when the main interaction is located in a physical arena.

It is important to note that the digital component does not affect the input activity but facilitates a substitution of the competition and digitalization of the sporting activities and the arena, which becomes physically distributed (e.g. through the Zwift cycling platform). Because of the one-to-one relationship between the physical input and the digitally mediated output, little room is given to the potential influence of second and third order actors. While technically possible, the governing of this sport configuration focuses on maintaining the original nature of the physical activity. Instead of transforming the input activity, digital replication can be observed in this cluster of sport to engage in cognitive and preparatory activities (e.g., recruitment via simulator races or testing car set-ups in simulated physical environments) or to create a new form of engagement activities for spectators outside of the competition. As to the latter, cycling amateurs can compare their own performance to that of the professionals in the digital arena in realtime (e.g. the online L'Etape du Tour mass participation event on Zwift).

\subsection{Digitally translated sport}

The final cluster of sport configurations is the digitally translated sport, to which eSport belongs. The main difference between digitally replicated sport 
and this cluster is that in the former, performance is replicated one-to-one in a digital arena, while in the latter, this is not the case. Even the eSport categories that simulate a physical sport (e.g., FIFA football), players compete through controllers. This physical input activity of manipulating the controller with speed and dexterity is translated into a virtually realized/computed skill (e.g. football, shooting, spellcasting). Therefore, the physical input is translated into performance of a different nature in a digital arena. In other words, the core of the competition is not based on traditional physical skills anymore, but motion skills such as dexterity. The translation of the skill is the key distinguishing factor between digitally replicated and translated sport, as sport performed in full simulator set-ups (e.g. racing cockpits in motorsport) do not show such translation and are instead replicating the skill. Full motion simulation eSport (e.g. virtual cycling, motorsport sim racing) therefore count as digitally replicated sport, forming the exception to eSport as digitally translated sport. A further distinction is that in translated sport, one person may control multiple "players" or avatars in-game.

In this cluster, all interactions are fully digital and correspondingly located in a digital arena. It is worth noting that in many cases, the facilitation of the competition, i.e. refereeing, is for the most part computed through game mechanics and embedded in the digital arena.

This cluster of sport drastically changes the perception of the physical activity performed by first order actors, tying right into the discussion of what constitutes a physical activity. Finally, this cluster is again characterized by full openness to second and third order actors. Their influence does not manifest as physical influence, but as influence on the digitally translated performance.

\section{Revisiting the configuration of sport}

\subsection{Intertwining relationships between sporting activities}

Across all four configurations, the various in-game activities remain as the central input to create the sport performance output. The rules of the game provide a structure according to which the different input activities interact and relate to each other in order to construct the performance output. However, where previously only physical activities constructed the performance output, digitalization has introduced digital forms of sporting activities. These digital activities can manifest as evolutions of traditional activities, e.g. from post-game to real-time analyses of performance data, or they can manifest as new activities, such as the popularity voting in the Formula E FanBoost mechanism. As with the traditional sporting activities, the distinction between digital ingame activities and digital preparatory activities can be made. Digital activities such as health or recruitment analytics fall into the category of digital preparatory activities. Digital in-game activities on the other hand include real-time data analytics for strategizing, remote adjustment of equipment settings, unlocking of performance boosts based on online voting, or computing and simulation of performances and competition environments.

We conceptualize the interplay between the physical and digital activities as different intertwining relationships. Across the four identified clusters of configurations, varying degrees of intertwining can be observed. The differences in the intertwining configurations depend on the role and position of the digital activities (and components in a broader sense), which will in turn characterize the competition.

The intertwined relationships, across all physicaldigital hybrid sport configurations, display the four effects of intertwined relationships identified by Robey et al. [28]. In all four configurations, intertwining of the physical and digital displays reciprocal relationships. However, the intertwined relationships for each configuration appear to be dominated by one of the three other effects: complementarity, synergy, and reinforcement.

Digitally supported sport is characterized by a relationship where the physical component directly creates the output without being modified in nature. The digital component exists in parallel to reinforce and support non-essential elements. This results in a potentially higher performance without having affected the nature/core of the competition.

Digitally augmented sport is characterized by the synergistic interaction of physical and digital components, which results in a new form of outcome exceeding the individual components. Both components become integral parts of the competition.

Digitally replicated sport displays an intertwined relationship where the physical component is directly represented or replicated in the digital realm. This form of intertwining benefits from complementary strengths of the components by maintaining the nature/core of the physical competition while being performed in the digital realm. The digital component fully mediates the physical input and virtually simulates the physical arena (i.e., the environment of the competition. It is important to note how the digital components are intentionally embedded in the competition context in a way as to not give new meaning, direction and function to the core of the competition [22]. 
Finally, in digitally translated sport, the intertwined relationship similarly relates to the transfer of the physical component to the digital realm. However, this translation radically changes the nature of the original input (rather than simply replicating the physical activities as in the previous category). The digital component realizes and simultaneously governs the performance and is at the center of the competition. This form of intertwining strongly relates to the synergy effect, as it combines physical and digital components to create something new, exceeding the individual components.

\subsection{Emergence of the digital arena}

In line with our delineation above, the arena is understood as the location where the competitive ingame activities interact. Digital technologies introduce a possibility to extend or even substitute the physical arena. We observe that digital technologies enable the distributed performance of competitive sporting activities (e.g. the physical distribution of athletes in virtual bike races) where the interactions between activities are digitally facilitated [25]. Therefore, the understanding of the digital arena encompasses the locations where these digital sporting activities are performed or interact, such as digital platforms, virtual environments, or social media engagement platforms.

As shown in Table 1, physical-digital hybrid sport can be differentiated according to whether the main interaction occurs as a physical or virtual interaction [25], i.e. in the physical or digital arena.

The element of environmental unpredictability that is characteristic in the physical arena is not equivalently present in the digital arena. In cases where the digital arena serves not as an expansion to the physical realm but substitutes it, this unpredictability therefore would have to be simulated to achieve the same effect.

\subsection{Enabling new forms of actor(s) influence}

We observe that digital technologies are changing how the different orders of actors can influence the performed activity. The influence of athlete(s) as first order actor(s) remains unchanged as they physically perform the core activity. However, the intertwining of the physical core activity with digital activities introduces an opening for additional participants. Equipment embedded with computing capabilities enables, for example, remote control access or the conditional unlocking of abilities or resources. As a consequence, second order actors can directly implement their cognitive input (e.g. changing engine settings via remote control) and third order actors can influence/trigger conditions that affect the core activities (e.g. FanBoost). This implies that digital technologies facilitate the possibility for second and third order actors to have a direct influence on the performance of the core activity.

\subsection{Updated framework of sport configurations}

In Figure 2, we revise the framework of traditional sport configuration (see Figure 1) to account for the impact of digitalization on the different dimensions. Firstly, technologies digitalize existing sporting activities and introduce new digital activities that intertwine with physical ones to create new forms of competition and sport performance. Secondly, the sporting arena as location of the main interaction is no longer limited to a specific physical location but can be digital and distributed. Finally, digital technologies facilitate new forms of influence allowing all three orders of actors to participate.

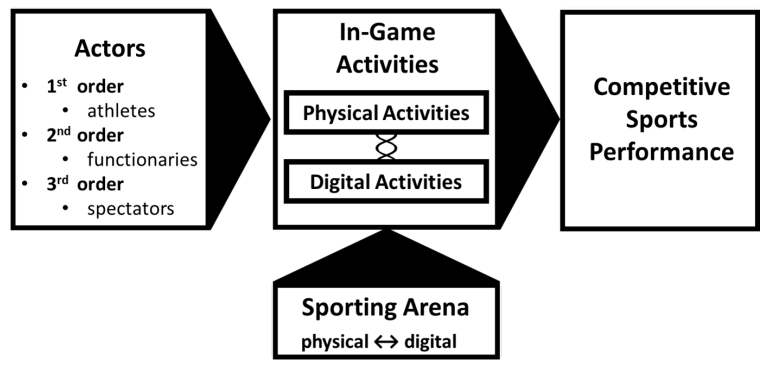

Figure 2. Physical-digital hybrid configuration of sport

\section{Implications}

\subsection{Implications for IS research}

In this paper, we have focused on the sport context and investigated the different intertwining relationships of digital-physical hybrid activities. Specifically, we utilize such context to illustrate and conceptualize the interdependence of digital and physical components and to introduce an ontology of hybrid configurations.

This initial study, which is part of a larger research project on sports digitalization, extends our understanding of the fusing of the digital and physical worlds $[3,45]$. In the intertwining of components that we conceptualized, physical and digital elements are jointly shaping the physical environments and reality.

By examining the various sport configurations, we have observed that what constitutes sport places an inherent physicality at its heart. The fundamentality of sport being performed through physical activities delimits potential digital innovations, since if it were to be fully digital, it would not be considered sport 
anymore. In this regard, the sport context proves to be a fertile ground to further investigate how digital technologies interact with such unique physical settings.

Furthermore, our conceptualizations provide some preliminary insights into the role of a context's physical aspects on the leverage points of digital innovation [22]. On the one hand, we can for instance observe how the sport context creates a boundary to the sensible digitalization of activities. Some aspects of sport are intentionally not digitalized to maintain the inherent physical sporting nature. This reinforces the notion that in certain contexts, uniquely physical value can exist and cannot be captured through digital means [46]. On the other hand, we observe that sport configurations that engage in replication and augmentation rely heavily on pre-existing equipment, e.g. cars or bikes. In these instances, the engineered transmission of the physical activity allows us to digitally measure an input as well as to augment an output. Specifically, we see purposeful and sensible digitalized activities in situations where functionality has already been inscribed into a material bearer [10]. These observations suggest that sensible digitalization of real-world phenomena might prove especially challenging in situations of non-material, purely physical interactions - imagine for instance the physical effects of a hit in boxing.

\subsection{Implications for Sport Management}

From a sport management perspective, our work provides an initial framework for conceptualizing the physical-digital hybrid nature of sport. Understanding the potential role and positioning of digital components in sport configurations provides insight and guidance on how technologies interact with the existing structure of a given sport. Our conceptualization has revealed that the position of the digital component in sporting activities determines to what extent the activities can be open to second or third order actors, due to the fact that only the digital component is malleable and hence can be connected to actors that were previously not directly involved in performing the sport. Further, sport that involves hardware (e.g., car, bike, etc.) offers more opportunities for digitalization in terms of intertwining digital and physical components. Therefore, sport management can focus on the introduction and positioning of the digital component to understand the potential of utilizing digital means for training purposes or to enhance fan engagement.

Another notable observation concerns non-human actors. Looking at eSport, we can already observe activities performed by computed machine actors. How non-human actors engage in competitive activity in the future and whether this will still be considered "sport" can be further discussed. One could for instance imagine a fifth cluster of sport configurations that could be referred to as digitally transformed sport. An example for such a configuration could be autonomous drone racing [33], in which the competitive physical activity is not performed by a human actor, but instead by a machine actor.

\section{References}

[1] Adi Gaskell, "Is The Virtual Tour De France The Next Stage In The Evolution Of ESports?", Forbes, 2020.accessed July 8, $2020 \mathrm{https}: / / w w w . f o r b e s . c o m / s i t e s / a d i g a s k e l l / 2020 /$ 07/03/is-the-virtual-tour-de-france-the-next-stage-in-theevolution-of-esports/\#3589f76d7f73

[2] Anagnostopoulos, C., P. Parganas, S. Chadwick, and A. Fenton, "Branding in pictures: using Instagram as a brand management tool in professional team sport organisations", European Sport Management Quarterly 18(4), 2018, pp. 413-438.

[3] Baskerville, R.L., M.D. Myers, and Y. Yoo, "Digital First: The Ontological Reversal and New Challenges for Information Systems Research", MIS Quarterly 44(2), 2020, pp. 509-523.

[4] Caya, O., and A. Bourdon, "A Framework of Value Creation from Business Intelligence and Analytics in Competitive Sports", 49th Hawaii International Conference on System Sciences, (2016), 1061-1071.

[5] Cunningham, G.B., S. Fairley, L. Ferkins, et al., "eSport: Construct specifications and implications for sport management", Sport Management Review 21(1), 2018, pp. $1-6$.

[6] Davenport, T.H., Analytics in Sports: The New Science of Winning, 2014.

[7] Decoster, C., and W. Standaert, "Collective Intelligence in the Context of Sports Performance Evaluation", $A C M$ Collective Intelligence, (2020).

[8] Elsam, S., "Interview: Tim Reichert, FC Schalke 04 Esports - Traditional Teams Need to Approach Esports in a 'Bigger Way"', 2018.accessed July 8, $2020 \mathrm{https://}$ esportsobserver.com/tim-reichert-schalke-04-interview/ [9] Faulkner, P., and J. Runde, "Technology Objects,Social Positions and The Tranformational Model of Social Activity", MIS Quarterly 37(3), 2013, pp. 803-818. [10] Faulkner, P., and J. Runde, "Theorizing the digital object”, MIS Quarterly 43(4), 2019, pp. 1279-1302. [11] Funk, D.C., A.D. Pizzo, and B.J. Baker, "eSport management: Embracing eSport education and research opportunities", Sport Management Review 21(1), 2018, pp. $7-13$.

[12] Geurin, A.N., and L.M. Burch, "User-generated branding via social media: An examination of six running brands", Sport Management Review 20(3), 2017, pp. 273284.

[13] Gruettner, A., "What we know and what we do not know about digital technologies in the sports industry", 25 th Americas Conference on Information Systems, (2019). [14] Hallmann, K., and T. Giel, "eSports - Competitive sports or recreational activity?", Sport Management Review 
21(1), 2018, pp. 14-20

[15] Hamilton, T., "Bundesliga suggests home advantage a thing of the past in empty stadiums", ESPN, 2020.accessed July 8, 2020 https://www.espn.com/soccer/germanbundesliga/story/4107639/bundesliga-suggests-homeadvantage-a-thing-of-the-past-in-empty-stadiums [16] Hilvoorde, I. van, and N. Pot, "Embodiment and fundamental motor skills in eSports", Sport, Ethics and Philosophy 10(1), 2016, pp. 14-27.

[17] Jarvenpaa, S.L., and W. Standaert, "Digital Probes as Opening Possibilities of Generativity", Journal of the Association for Information Systems 19(10), 2018, pp. 9821000.

[18] Jenny, S.E., R.D. Manning, M.C. Keiper, and T.W. Olrich, "Virtual(ly) Athletes: Where eSports Fit Within the Definition of 'Sport", Quest 69(1), 2017, pp. 1-18. [19] Leonardi, P.M., "Digital materiality? How artifacts without matter, matter", First Monday 15(6), 2010. [20] Li, B., S.W. Dittmore, O.K.M. Scott, W. Lo, and S. Stokowski, "Why we follow: Examining motivational differences in following sport organizations on Twitter and Weibo", Sport Management Review 22(3), 2019, pp.335-347. [21] Loy, J.W., "The Nature of Sport: A Definitional Effort", Quest 10(1), 1968, pp. 1-15.

[22] Lyytinen, K., "Innovation Logics in the Digital Era: Systemic Review of Emerging Digital Innovation Regime", working paper, 2020.

[23] Marino, A., P. Aversa, L. Mesquita, and J. Anand, "Driving performance via exploration in changing environments: Evidence from formula one racing", Organization Science 26(4), 2015, pp. 1079-1100. [24] Miah, A., Sport 2.0: Transforming Sports for a Digital World, Massachusettes Institute of Technology, Cambridge, Massachusetts; London, England, 2017.

[25] Overby, E., "Process Virtualization Theory and the Impact of Information Technology", Organization Science 19(2), 2008, pp. 277-291.

[26] Overby, E., S.A. Slaughter, and B. Konsynski, "Research Commentary - The Design, Use, and Consequences of Virtual Processes", Information Systems Research 21(4), 2010, pp. 700-710.

[27] Ringuet-Riot, C.J., A. Hahn, and D.A. James, "A structured approach for technology innovation in sport", Sports Technology 6(3), 2013, pp. 137-149.

[28] Robey, D., K.S. Schwaig, and L. Jin, "Intertwining material and virtual work", Information and Organization 13(2), 2003, pp. 111-129.

[29] Seo, H., S. Kim, and S. Yoon, "Moderating effects of multi-dimensional organizational commitment between knowledge sharing and Innovative behavior among instructors in sport centers", Korean Journal of Sports Management 18(1), 2013, pp. 45-58.

[30] Shah, F.A., M. Kretzer, and A. Mädche, "Designing an analytics platform for professional sports teams", 36th International Conference on Information Systems, (2015), 120.

[31] Smith, A.C.T., and B. Stewart, "The special features of sport: A critical revisit”, Sport Management Review 13(1), 2010, pp. 1-13.

[32] Smith, E.E., and J.D. Groetzinger, "Do Fans Matter?

The Effect of Attendance on the Outcomes of Major League
Baseball Games", Journal of Quantitative Analysis in Sports 6(1), 2010.

[33] Standaert, W., "Digital Growth Strategies at Drone Racing League", Journal of Information Technology Teaching Cases,

[34] Standaert, W., and S.L. Jarvenpaa, "Formula E: Next generation motorsport with next generation Fans", 37th International Conference on Information Systems, (2016), 116.

[35] Stavros, C., M.D. Meng, K. Westberg, and F. Farrelly, "Understanding fan motivation for interacting on social media", Sport Management Review 17(4), 2014, pp. 455-469. [36] Suits, B., "The element of sport", In W. Morgan, ed., Ethics in sport. Human Kinetics, Champaign, IL, 2007, 9-19. [37] Tan, F., J. Hedman, and X. Xiao, "Beyond 'Moneyball' to Analytics Leadership in Sports: An Ecological Analysis of FC Bayern Munich's Digital Transformation", 23rd

Americas Conference on Information Systems, (2017), 1-5.

[38] Tjønndal, A., "Sport innovation: developing a typology", European Journal for Sport and Society 14(4), 2017, pp. 291-310.

[39] Troilo, M., A. Bouchet, T.L. Urban, and W.A. Sutton, "Perception, reality, and the adoption of business analytics: Evidence from North American professional sport organizations", Omega 59, 2016, pp. 72-83.

[40] van de Ven, N., "Supporters are not necessary for the home advantage: Evidence from same-stadium derbies and games without an audience.", Journal of Applied Social Psychology 41(12), 2011, pp. 2785-27858.

[41] Wagner, M., "Competing in metagame gamespace: eSport as the first professional-sized computer metagames", In F. von Borries, S.P. Walz, M. Bèottger, D. Davidson, H. Kelley and J. Keucklich, eds., Space time play: Synergies between computer games, architecture and urbanism: The next level. Springer, New York, 2007, 182-185.

[42] Wright, M.B., "50 Years of OR in Sport", The Journal of the Operational Research Society 60, 2009, pp. S161-S168. [43] Xiao, X., F. Ter Chian Tan, E.T.K. Lim, et al., "Sports Digitalization: An Overview and A Research Agenda", 38th International Conference on Information Systems, (2017), 121.

[44] Yan, G., N.M. Watanabe, S.L. Shapiro, M.L. Naraine, and K. Hull, "Unfolding the Twitter scene of the 2017 UEFA Champions League Final: social media networks and power dynamics", European Sport Management Quarterly 19(4), 2019, pp. 419-436.

[45] Yoo, Y., "Computing in Everyday Life: A Call for Research on Experiential Computing”, MIS Quarterly 34(2), 2010, pp. 213-231.

[46] Yoo, Y., and J. Euchner, "Digital First Thinking for Industrial Companies: An Interview with Youngjin Yoo", Research Technology Management 63(3), 2020, pp. 12-18. [47] Yoo, Y., O. Henfridsson, and K. Lyytinen, "Research Commentary-The New Organizing Logic of Digital Innovation: An Agenda for Information Systems Research", Information Systems Research 21(4), 2010, pp. 724-735. [48] "materiality", OED Online, 2020.accessed July 12, 2020 www.oed.com/view/Entry/114928

[49] "physicality", OED Online, 2020.accessed July 12, 2020 www.oed.com/view/Entry/143124 